\title{
A Nonequilibrium Evolution Criterion for Electromagnetic Bodies
}

\author{
Eugenia Radzikowska ${ }^{1}$, Romuald Kotowski ${ }^{1,2}$, Wolfgang Muschik ${ }^{3}$ \\ ${ }^{1}$ Institute of Fundamental Technological Research, Polish Academy of Sciences, Warsaw, \\ Poland \\ ${ }^{2}$ Polish-Japanese Institute of Information Technology, Warsaw, Poland \\ ${ }^{3}$ Institut für Theoretische Physik, Technische Universität Berlin, Berlin, Germany \\ Communicated by G. A. Maugin, Paris, France \\ Registration Number 867
}

\begin{abstract}
In this paper the micropolar medium exposed to the action of mechanical, thermal and electromagnetic fields is considered. The generalization of the global evolution criterion of nonequilibrium thermodynamics of micropolar media in the case of nonvanishing electromagnetic fields is derived. Interaction with the environment is modeled by the black-body radiation. Application of the criterion to liquid crystals in equilibrium state is given and the complete set of fundamental equations is derived.
\end{abstract}

\section{Introduction}

The possibility of the realization of a given physical process in the realm of everyday experience is a problem which has been studied for many years. The classical formulation can be found e.g. in [1-4]. The more modern approach, taking into account recent achievements of nonequilibrium thermodynamics of irreversible processes was given in [5] and [6]. The present paper is the generalization of previous results for the case of electromagnetic interactions. We assume that the medium has a micropolar structure. As an application example of the obtained criterion, the liquid crystal in equilibrium is considered. In this paper we use the following tensors product convention: if $\boldsymbol{A}$ is a tensor of order $n,(n=1,2, \ldots)$ with components $A^{i, j, \ldots, m, n}$ and $\boldsymbol{B}$ is a tensor of order $l,(l=1,2, \ldots)$, with components $B_{r, s, \ldots, k, l}$ then the product $\boldsymbol{A} \cdot \boldsymbol{B}$ is the tensor of order $n+l-2$ with components $A^{i, j, \ldots, m, n} B_{n, s, \ldots, k, l}$ and the double product $\boldsymbol{A}: \boldsymbol{B}$ is the tensor of order $n+l-4$ with components $A^{i, j, \ldots, m, n} \boldsymbol{B}_{m, n, \ldots, k, l}$.

\section{Formulation of the Problem}

Starting points are the first two laws of thermodynamics, which we formulate in the following way [7-10]: 
- First law of thermodynamics (global energy balance)

$$
\begin{aligned}
& \frac{D}{D t} \int_{\mathscr{G}(t)}\left[\rho \epsilon+\frac{1}{2} \rho \boldsymbol{v} \cdot \boldsymbol{v}+\frac{1}{2} \rho \boldsymbol{\sigma} \cdot \boldsymbol{\omega}+\frac{1}{2}\left(\varepsilon_{o} \boldsymbol{E} \cdot \boldsymbol{E}+\frac{1}{\mu_{o}} \boldsymbol{B} \cdot \boldsymbol{B}\right)\right] d V \\
& \quad-\oint_{\partial \mathscr{G}(t)} \boldsymbol{n} \cdot\left[\left(\boldsymbol{t}+{ }_{\mathbf{M}} \boldsymbol{t}+\boldsymbol{v} \otimes \boldsymbol{G}\right) \cdot \boldsymbol{v}+\boldsymbol{m} \cdot \boldsymbol{\omega}+\boldsymbol{q}-\mathscr{S}\right] d A \\
& \quad-\int_{\mathscr{G}(t)} \rho(\tilde{\boldsymbol{f}} \cdot \boldsymbol{v}+\tilde{\boldsymbol{l}} \cdot \boldsymbol{\omega}+\tilde{h}) d V=0,
\end{aligned}
$$

- Second law of thermodynamics (global entropy inequality)

$$
\frac{D}{D t} \int_{\mathscr{G}(t)} \rho \eta d V-\oint_{\partial \mathscr{G}(t)} \boldsymbol{n} \cdot \boldsymbol{s} d A-\int_{\mathscr{G}(t)} \rho \tilde{b} d V \geq 0
$$

We have used here the following notation ([5], [7], [11]): $\rho$ - mass density, $\boldsymbol{q}$ - the heat vector (directed opposite to the flux of heat), $\mathscr{S}-$ the Poynting vector, $\boldsymbol{G}-$ the electromagnetic momentum vector, $\mathbf{M} t-$ the Maxwell stress tensor, $\tilde{h}-$ the specific energy source, $\epsilon$ - the specific internal energy, $s-$ the influx of entropy through the surface of the body, $\eta$ - the specific entropy, $\tilde{b}$ - the specific entropy source, $t-$ the stress tensor, $\boldsymbol{m}$ - the couple stress tensor, $\boldsymbol{\sigma}-$ the specific spin vector, $\boldsymbol{v}-$ the material velocity, $\omega-$ the angular velocity vector, $\tilde{\boldsymbol{f}}-$ the specific body forces vector, $\tilde{\boldsymbol{l}}-$ the imposed specific body couple vector, $\boldsymbol{n}$ - the exterior unit normal (see Fig. 1).

Let us make some remarks on the second law of thermodynamics (2). It is generally accepted [3] that the change of entropy $d S$ can be split into two parts: the entropy production $d_{i} S$ caused by the changes (irreversible processes) inside the system, and

$\mathcal{G}^{*}(t)$

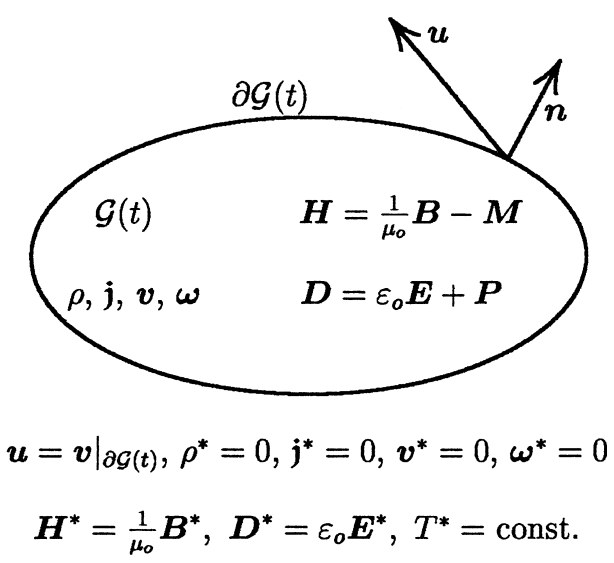

Fig. 1. A closed discrete system $\mathscr{G}(t)$ in a source-free equilibrium environment $\mathscr{G}^{*}(t)$ of blackbody radiation of the temperature $T^{*}$. 
the production of entropy $d_{e} S$ caused by the interaction of the body with the external world, i.e.

$$
d S=d_{i} S+d_{e} S
$$

In this terminology we have

$$
\begin{aligned}
& d S \doteq \frac{D}{D t} \int_{\mathscr{G}(t)} \rho \eta d V \\
& d_{e} S \doteq \oint_{\partial \mathscr{G}(t)} \boldsymbol{n} \cdot \boldsymbol{s} d A+\int_{\mathscr{G}(t)} \rho \tilde{b} d V .
\end{aligned}
$$

The entropy $d_{e} S$ can be supplied to the system through the boundary and by the internal body sources. The entropy production $d_{i} S$ is never negative: this is the second law of thermodynamics.

The local forms of the first (1) and the second (2) laws of thermodynamics are:

- Local energy balance

$$
\begin{aligned}
\rho \dot{\epsilon}-\boldsymbol{t} & : \boldsymbol{a}-\boldsymbol{m}^{T}: \boldsymbol{b}-\nabla \cdot \boldsymbol{q}-\rho \tilde{h} \\
& -\mathscr{E} \cdot[\mathscr{J}+\dot{\boldsymbol{P}}+\boldsymbol{P}(\nabla \cdot \boldsymbol{v})]+\mathscr{M} \cdot \dot{\boldsymbol{B}}+(\boldsymbol{P} \times \mathscr{E}+\mathscr{M} \times \boldsymbol{B}) \cdot \boldsymbol{\omega}=0,
\end{aligned}
$$

with the boundary condition on $\partial \mathscr{G}(t)$ :

$$
\begin{aligned}
\boldsymbol{n} \cdot & \llbracket\left(\rho \epsilon+\frac{1}{2} \rho \boldsymbol{v} \cdot \boldsymbol{v}+\frac{1}{2} \rho \boldsymbol{\sigma} \cdot \boldsymbol{\omega}+\frac{1}{2} \varepsilon_{o} \boldsymbol{E} \cdot \boldsymbol{E}+\frac{1}{2 \mu_{o}} \boldsymbol{B} \cdot \boldsymbol{B}\right)(\boldsymbol{v}-\boldsymbol{u}) \\
& -\left(\boldsymbol{t}+{ }_{\mathbf{M}} \boldsymbol{t}+\boldsymbol{u} \otimes \boldsymbol{G}\right) \cdot \boldsymbol{v}-\boldsymbol{m} \cdot \boldsymbol{\omega}-(\boldsymbol{q}-\mathscr{S}) \rrbracket=0
\end{aligned}
$$

- Local entropy inequality

$$
\rho \dot{\eta}-\nabla \cdot \boldsymbol{s}-\rho \tilde{b} \geq 0
$$

with the boundary condition on $\partial \mathscr{G}(t)$ :

$$
\boldsymbol{n} \cdot \llbracket \rho \eta(\boldsymbol{v}-\boldsymbol{u})-\boldsymbol{s} \rrbracket \geq 0 .
$$

Here $\boldsymbol{u}$ is the velocity of the boundary surface, $\boldsymbol{a}$ and $\boldsymbol{b}$ are the deformation rate tensors

$$
\boldsymbol{a} \doteq \nabla \otimes \boldsymbol{v}-\boldsymbol{\varepsilon} \cdot \boldsymbol{\omega}, \quad \boldsymbol{b}^{T} \doteq \nabla \otimes \boldsymbol{\omega}
$$

$\boldsymbol{P}$ and $\mathscr{M}$ are the polarization and magnetization vectors, respectively, which were defined e.g. in [7], [11] (the list of symbols is given at the end of the paper). The body is influenced by the electric field $\boldsymbol{E}=\boldsymbol{E}(\boldsymbol{x}, t)$ and the magnetic induction $\boldsymbol{B}=\boldsymbol{B}(\boldsymbol{x}, t)$. 
Equation (5) and inequality (7) have resulted in the localization process of the global thermodynamic laws [8-10] and are satisfied at every point of the micropolar continuum $\mathscr{G}(t)$. Analogously, the boundary conditions (6) and (8) are satisfied on any arbitrary discontinuity surface $\Sigma(t)$, sweeping the continuum $\mathscr{G}(t)$ and moving with the velocity $\boldsymbol{u}(\boldsymbol{x}, t)$. In the case we considered, the (micropolar continuum) body is situated in a vacuum filled by the thermal field of the black-body radiation, and the boundary conditions (6) and (8) on the boundary of the body $\partial \mathscr{G}(t)$ take the form

$$
\begin{aligned}
\boldsymbol{n} \cdot & {\left[\frac{1}{2}\left(\varepsilon_{o} \boldsymbol{E}^{+} \cdot \boldsymbol{E}^{+}+\frac{1}{\mu_{o}} \boldsymbol{B}^{+} \cdot \boldsymbol{B}^{+}\right) \boldsymbol{u}+\boldsymbol{q}^{+}-\mathscr{S}^{+}\right] } \\
& =-\boldsymbol{n} \cdot\left[\left(\boldsymbol{t}^{-}+\mathbf{M}^{-}+\boldsymbol{u} \otimes \boldsymbol{G}^{-}\right) \cdot \boldsymbol{v}^{-}+\boldsymbol{m}^{-} \cdot \boldsymbol{\omega}^{-}+\boldsymbol{q}^{-}-\mathscr{S}^{-}\right], \\
\boldsymbol{n} \cdot \llbracket \boldsymbol{s} \rrbracket \leq 0 &
\end{aligned}
$$

where $\llbracket F \rrbracket \doteq F^{+}-F^{-}$denotes the jump on the surface $\Sigma(t)$ between the exterior $F^{+}$ and the interior $F^{-}$values of the field $F$.

Liggieri et al. [12] described the effects of the electromagnetic field on the surface tension of liquids. The calculations were made for anisotropic fluid layers at local mechanical equilibrium.

The closed system $\mathscr{G}(t)\left(\boldsymbol{u}=\left.\boldsymbol{v}\right|_{\partial \mathscr{G}(t)}\right)$ is in contact by $\partial \mathscr{G}(t)$ with its environment $\mathscr{G}^{*}(t)$, which is a reservoir having the following properties for all times: the reservoir is source-free $\left(\rho^{*}=0, \mathbf{j}^{*}=0\right)$ and consists of black-body radiation of constant temperature $T^{*}$. This means that

$$
\nabla T^{*}=\mathbf{0}, \quad \frac{\partial T^{*}}{\partial t}=0
$$

The motion of $\mathscr{G}(t)$ is described by

$$
x^{k}=x^{k}(\boldsymbol{X}, t), \quad X^{K}=X^{K}(\boldsymbol{x}, t) .
$$

Here $\boldsymbol{X}$ is the initial position of the material point which is at time $t$ in the actual position $\boldsymbol{x}$. The internal degree of freedom of the micropolar medium obeys

$$
\xi^{k}=\chi_{K}^{k}(\boldsymbol{X}, t) \Xi^{K}, \quad \Xi^{K}=\chi_{k}{ }^{K}(\boldsymbol{x}, t) \xi^{k},
$$

for the rotational motion of the director $\Xi$ attached to the material point $\boldsymbol{X}$ [8], [9]. Relations in (14) are reciprocal and $\chi$, called sometimes the micromotion, is a proper orthogonal tensor $\left(\boldsymbol{\chi}^{-1}=\boldsymbol{\chi}^{T}\right.$, det $\left.\chi=1\right)$. Therefore,

$$
\begin{gathered}
x^{k}{ }_{{ }_{K}} X^{K},{ }_{l}=\delta_{l}^{k}, \quad X^{K},{ }_{k} x^{k},{ }_{L}=\delta_{L}^{K}, \\
\chi^{k}{ }_{K} \chi_{l}{ }^{K}=\delta_{l}^{k}, \quad \chi^{k}{ }_{L} \chi_{k}{ }^{K}=\delta_{L}^{K} .
\end{gathered}
$$

In order to define the energy of a micropolar material, we need the following deformation tensors [8], [9]: 
- the Cosserat deformation tensors $\mathfrak{C}_{K L}$ and $\mathfrak{c}_{k l}$

$$
\mathfrak{c}_{K L} \doteq x^{k},{ }_{K} \chi_{k L}, \quad \mathfrak{c}_{k l} \doteq X^{K},{ }_{k} \chi_{l K}
$$

- the wryness tensors $\Gamma_{K L}$ and $\gamma_{k l}$

$$
\Gamma_{K L} \doteq \frac{1}{2} \varepsilon_{K M N} \chi^{k M},{ }_{L} \chi_{k}{ }^{N}, \quad \gamma_{l k} \doteq \frac{1}{2} \varepsilon_{l m n} \chi^{m K} \chi_{K}^{n},{ }_{k} .
$$

Hence, we arrive at the following equations

$$
\begin{aligned}
& \gamma_{l k}=X^{K},_{k} \chi_{l}{ }^{L} \Gamma_{L K}, \\
& a_{k l}=X^{K},{ }_{k} \chi_{l}{ }^{L} \dot{\boldsymbol{C}}_{K L}, \\
& b_{l k}=X^{K},{ }_{k} \chi_{l}{ }^{L} \dot{\Gamma}_{L K} .
\end{aligned}
$$

For the purpose of our calculations, we assume that the body forces $\tilde{f}$ are potential forces,

$$
\tilde{\boldsymbol{f}}=-\nabla \tilde{g}, \quad \frac{\partial \tilde{g}}{\partial t}=0
$$

i.e. $\tilde{g}=\tilde{g}(x)$. The body moments $\tilde{\boldsymbol{l}}$ can be represented by an analogous function $\tilde{\lambda}=\tilde{\lambda}(\xi)$, so that

$$
\tilde{\boldsymbol{l}}=\frac{d \tilde{\lambda}}{d \xi} \times \boldsymbol{\xi}
$$

is valid. According to (14), $\boldsymbol{\xi}$ is the director attached to every point $\boldsymbol{x}$ of the micropolar body. If $\omega$ is the angular velocity vector defined by

$$
\dot{\xi}=\omega \times \xi
$$

then

$$
\tilde{\boldsymbol{l}} \cdot \boldsymbol{\omega}=-\dot{\tilde{\lambda}}
$$

Now we apply the procedure presented in the paper of Muschik and Papenfuss [5]. We multiply (2) by the constant exterior temperature $T^{*}$ and find the difference with (1), which gives the following thermodynamic inequality

$$
\begin{aligned}
& \frac{D}{D t} \int_{\mathscr{G}(t)}\left[\rho\left(T^{*} \eta-\epsilon-\frac{1}{2} \boldsymbol{v} \cdot \boldsymbol{v}-\frac{1}{2} \boldsymbol{\sigma} \cdot \boldsymbol{\omega}\right)-\frac{1}{2}\left(\varepsilon_{o} \boldsymbol{E} \cdot \boldsymbol{E}+\frac{1}{\mu_{o}} \boldsymbol{B} \cdot \boldsymbol{B}\right)\right] d V \\
& \quad+\oint_{\partial \mathscr{G}(t)} \boldsymbol{n} \cdot\left[\left(\boldsymbol{t}+\mathbf{M}^{\boldsymbol{t}}+\boldsymbol{v} \otimes \boldsymbol{G}\right) \cdot \boldsymbol{v}+\boldsymbol{m} \cdot \boldsymbol{\omega}+\boldsymbol{q}-\mathscr{S}-T^{*} \boldsymbol{s}\right] d A \\
& \quad+\int_{\mathscr{G}(t)} \rho\left(\tilde{\boldsymbol{f}} \cdot \boldsymbol{v}+\tilde{\boldsymbol{l}} \cdot \boldsymbol{\omega}+\tilde{h}-T^{*} \tilde{b}\right) d V \geq 0 .
\end{aligned}
$$


It was shown in [5] that the inequality

$$
\oint_{\partial \mathscr{G}(t)} \boldsymbol{n} \cdot\left(T^{*} \boldsymbol{s}-\boldsymbol{q}\right) d A \geq 0
$$

always holds. It was assumed, moreover, that the process is sourceless, i.e. that $\tilde{h}=0$ and that $\tilde{b}=0$, too. That was the sufficient condition to obtain the evolution criterion. It is seen from (23) that we can weaken the Muschik and Papenfuss' assumption by writing that the following inequality holds

$$
\int_{\mathscr{G}(t)} \rho\left(T^{*} \tilde{b}-\tilde{h}\right) d V+\oint_{\partial \mathscr{G}(t)} \boldsymbol{n} \cdot\left(T^{*} \boldsymbol{s}-\boldsymbol{q}\right) d A \geq 0,
$$

which is valid for certain specific applied sources only. Let us remark that in thermodynamics of continuous media it is usually assumed that [10]

$$
\boldsymbol{s}=\frac{1}{T} \boldsymbol{q}+\boldsymbol{s}^{\prime}, \quad \text { and } \quad T=\frac{\tilde{h}}{\tilde{b}},
$$

where $T=T(\boldsymbol{x}, t)$ is the absolute temperature of the body. The function $\boldsymbol{s}^{\prime}$ is an excess over the classical value $\boldsymbol{q} / T$ of the entropy flux which is different from zero e.g. for mixtures and complex materials (usually $\left.\boldsymbol{s}^{\prime}=\mathbf{0}\right)^{1)}$.

Taking into account the assumptions (19), (22) and (25) transform (23) to

$$
\begin{aligned}
& \frac{D}{D t} \int_{\mathscr{G}(t)}\left[\rho\left(T^{*} \eta-\epsilon-\frac{1}{2} \boldsymbol{v} \cdot \boldsymbol{v}-\frac{1}{2} \boldsymbol{\sigma} \cdot \boldsymbol{\omega}-\tilde{g}-\tilde{\lambda}\right)\right. \\
& \left.\quad-\frac{1}{2}\left(\varepsilon_{o} \boldsymbol{E} \cdot \boldsymbol{E}+\frac{1}{\mu_{o}} \boldsymbol{B} \cdot \boldsymbol{B}\right)\right] d V \\
& \quad+\oint_{\partial \mathscr{G}(t)} \boldsymbol{n} \cdot\left[\left(\boldsymbol{t}+{ }_{\mathbf{M}} \boldsymbol{t}+\boldsymbol{v} \otimes \boldsymbol{G}\right) \cdot \boldsymbol{v}+\boldsymbol{m} \cdot \boldsymbol{\omega}-\mathscr{S}\right] d A \geq 0 .
\end{aligned}
$$

It follows from the boundary condition (10) that

$$
\begin{aligned}
& \oint_{\partial \mathscr{G}(t)} \boldsymbol{n} \cdot\left[\left(\boldsymbol{t}+{ }_{\mathbf{M}} \boldsymbol{t}+\boldsymbol{v} \otimes \boldsymbol{G}\right) \cdot \boldsymbol{v}+\boldsymbol{m} \cdot \boldsymbol{\omega}-\mathscr{S}\right] d A \\
& =\oint_{\partial \mathscr{G}(t)} \boldsymbol{n} \cdot\left\{\frac{1}{2}\left(\varepsilon_{o} \boldsymbol{E}^{*} \cdot \boldsymbol{E}^{*}+\frac{1}{\mu_{o}} \boldsymbol{B}^{*} \cdot \boldsymbol{B}^{*}\right) \boldsymbol{u}-\frac{1}{\mu_{o}} \boldsymbol{E}^{*} \times \boldsymbol{B}^{*}+\llbracket \boldsymbol{q} \rrbracket\right\} d A .
\end{aligned}
$$

Now we assume that $\boldsymbol{E}^{*}, \boldsymbol{B}^{*}$ are the continuous fields described in $\mathscr{G}(t) \cup \mathscr{G}^{*}$ satisfying the sourceless Maxwell equations in $\mathscr{G}(t)$

$$
\nabla \times \boldsymbol{E}^{*}+\boldsymbol{B}_{, t}^{*}=\mathbf{0}, \quad \frac{1}{\mu_{o}} \nabla \times \boldsymbol{B}^{*}-\varepsilon_{o} \boldsymbol{E}_{, t}^{*}=\mathbf{0},
$$

\footnotetext{
${ }^{1)}$ Here we want to avoid introducing a temperature field of the non-equilibrium system $\mathscr{G}(t)$.
} 
Making use of Green-Gauss-Ostrogradski theorem and the transport theorem

$$
\frac{D}{D t} \int_{\mathscr{G}(t)} F d V=\int_{\mathscr{G}(t)} \frac{\partial F}{\partial t} d V+\oint_{\partial \mathscr{G}(t)} \boldsymbol{n} \cdot(F \boldsymbol{v}) d A,
$$

we obtain after simple calculations

$$
\begin{gathered}
\oint_{\partial \mathscr{G}(t)} \boldsymbol{n} \cdot\left[\frac{1}{2}\left(\varepsilon_{o} \boldsymbol{E}^{*} \cdot \boldsymbol{E}^{*}+\frac{1}{\mu_{o}} \boldsymbol{B}^{*} \cdot \boldsymbol{B}^{*}\right) \boldsymbol{u}-\frac{1}{\mu_{o}} \boldsymbol{E}^{*} \times \boldsymbol{B}^{*}\right] d A \\
=\frac{D}{D t} \int_{\mathscr{G}(t)} \frac{1}{2}\left(\varepsilon_{o} \boldsymbol{E}^{*} \cdot \boldsymbol{E}^{*}+\frac{1}{\mu_{o}} \boldsymbol{B}^{*} \cdot \boldsymbol{B}^{*}\right) d V .
\end{gathered}
$$

Muschik and Papenfuss [5] argue that

$$
\oint_{\partial \mathscr{G}(t)} \boldsymbol{n} \cdot \boldsymbol{s} d A \geq \frac{1}{T^{*}} \oint_{\partial \mathscr{G}(t)} \boldsymbol{n} \cdot \boldsymbol{q} d A
$$

It follows from the above inequality and from (2) that on the boundary $\partial \mathscr{G}$

$$
-\frac{1}{T^{*}} \boldsymbol{n} \cdot \llbracket \boldsymbol{q} \rrbracket \geq 0
$$

Hence we finally obtained the evolution criterion in the form

$$
\frac{D}{D t} \int_{\mathscr{G}(t)}\left[\rho\left(T^{*} \eta-\epsilon-\mathbf{k} e-\tilde{g}-\tilde{\lambda}\right)-\left(\mathbf{M} \epsilon-\mathbf{M} \epsilon^{*}\right)\right] d V \geq 0
$$

where $\rho_{\mathbf{K}} e$ is the kinetic energy of micropolar body, and

$$
\begin{aligned}
\mathbf{K} e & \doteq \frac{1}{2}(\boldsymbol{v} \cdot \boldsymbol{v}+\boldsymbol{\sigma} \cdot \boldsymbol{\omega}), \\
\mathbf{M} \epsilon & \doteq \frac{1}{2}\left(\varepsilon_{o} \boldsymbol{E} \cdot \boldsymbol{E}+\frac{1}{\mu_{o}} \boldsymbol{B} \cdot \boldsymbol{B}\right), \\
\mathbf{M} \epsilon^{*} & \doteq \frac{1}{2}\left(\varepsilon_{o} \boldsymbol{E}^{*} \cdot \boldsymbol{E}^{*}+\frac{1}{\mu_{o}} \boldsymbol{B}^{*} \cdot \boldsymbol{B}^{*}\right) .
\end{aligned}
$$

The evolution criterion (34) can be applied to determine the thermodynamic equilibrium conditions for deformable materials subjected to black-body radiation. In the next section we show the application of that formalism to liquid crystals.

\section{Equilibrium Conditions for Liquid Crystals}

We here use the Eringen approach to micropolar media. The basic equations describing liquid crystals subjected to the electromagnetic interactions inside the material body have the form [7], [11], [13]: 
- mass conservation law

$$
\frac{\partial \rho}{\partial t}+\nabla \cdot(\rho \boldsymbol{v})=0
$$

- microinertia conservation law

$$
\frac{D \mathbf{j}}{D t}+\omega \cdot \boldsymbol{\varepsilon} \cdot \mathbf{j}-\mathbf{j} \cdot \boldsymbol{\varepsilon} \cdot \boldsymbol{\omega}=\mathbf{0}
$$

- balance of momentum

$$
-\rho \dot{\boldsymbol{v}}+\nabla \cdot \boldsymbol{t}+{ }_{\mathbf{M}} \boldsymbol{f}+\rho \tilde{\boldsymbol{f}}=\mathbf{0},
$$

- balance of moment of momentum

$$
-\rho \dot{\boldsymbol{\sigma}}+\nabla \cdot \boldsymbol{m}+\boldsymbol{\varepsilon}: \boldsymbol{t}+{ }_{\mathbf{M}} \boldsymbol{l}+\rho \tilde{\boldsymbol{l}}=\mathbf{0},
$$

- Faraday law

$$
\frac{\partial \boldsymbol{B}}{\partial t}+\nabla \times \boldsymbol{E}=\mathbf{0}
$$

- magnetic flux conservation law (magnetic Gauss law)

$$
\nabla \cdot \boldsymbol{B}=0
$$

- Gauss electric law

$$
q_{f}-\nabla \cdot \boldsymbol{D}=0
$$

- Ampere law

$$
\frac{\partial \boldsymbol{D}}{\partial t}-\nabla \times \boldsymbol{H}+\boldsymbol{J}=\mathbf{0}
$$

where ${ }_{\mathbf{M}} \boldsymbol{f}=\nabla \cdot{ }_{\mathbf{M}} \boldsymbol{t}-\boldsymbol{G}{ }_{t},{ }_{\mathbf{M}} \boldsymbol{l}=\boldsymbol{\varepsilon}:{ }_{\mathbf{M}} \boldsymbol{t}^{T}, \boldsymbol{G} \doteq \varepsilon_{\mathrm{o}} \boldsymbol{E} \times \boldsymbol{B}$, and ${ }_{\mathbf{M}} \boldsymbol{t}$ is the Maxwell stress tensor defined by

$$
\mathbf{M} \boldsymbol{t} \doteq \boldsymbol{P} \otimes \mathscr{E}-\boldsymbol{B} \otimes \mathscr{M}+\varepsilon_{o} \boldsymbol{E} \otimes \boldsymbol{E}+\frac{1}{\mu_{o}} \boldsymbol{B} \otimes \boldsymbol{B}-(\mathbf{M} \epsilon-\mathscr{M} \cdot \boldsymbol{B}) \boldsymbol{I}
$$

According to the definition of the equilibrium state of the discrete system $\mathscr{G}(t)$ there exists such a global frame $\left(\boldsymbol{x}^{\prime}, t\right)$ [5] that all the material time derivatives (like velocity $\boldsymbol{v}\left(\boldsymbol{x}^{\prime}, t\right)$ and angular velocity $\left.\boldsymbol{\omega}\left(\boldsymbol{x}^{\prime}, t\right)\right)$, absolute temperature gradient $\nabla^{\prime} T\left(\boldsymbol{x}^{\prime}, t\right)$, heat vector $\boldsymbol{q}\left(\boldsymbol{x}^{\prime}, t\right)$, electric current $\mathscr{J}\left(\boldsymbol{x}^{\prime}, t\right)$, energy source $\tilde{h}\left(\boldsymbol{x}^{\prime}, t\right)$ and entropy supply $\tilde{b}\left(\boldsymbol{x}^{\prime}, t\right)$ vanish. All partial time derivatives vanish as well. Moreover, we assume that the axiom of material objectivity holds. 
It follows from the evolution criterion (34) that in the equilibrium state, the functional:

$$
L=\int_{\mathscr{G}^{\prime}}\left[\rho\left(T^{*} \eta-\epsilon-\tilde{g}-\tilde{\lambda}\right)-\left(\mathbf{M} \epsilon-\mathbf{M} \epsilon^{*}\right)\right] d V^{\prime},
$$

attains its maximum (because it is not decreasing), which means that

$$
\delta L=0
$$

We assume that vectors $\boldsymbol{E}\left(\boldsymbol{E}^{*}\right)$ and $\boldsymbol{B}\left(\boldsymbol{B}^{*}\right)$ can be expressed with the help of the vector potential $\boldsymbol{A}\left(\boldsymbol{x}^{\prime}\right)\left(\boldsymbol{A}^{*}\left(\boldsymbol{x}^{\prime}\right)\right)$ and the scalar potential $\varphi\left(\boldsymbol{x}^{\prime}\right)\left(\varphi^{*}\left(\boldsymbol{x}^{\prime}\right)\right)$ :

$$
\begin{aligned}
& \boldsymbol{E}=\nabla^{\prime} \varphi, \quad \boldsymbol{B}=\nabla^{\prime} \times \boldsymbol{A}, \\
& \boldsymbol{E}^{*}=\nabla^{\prime} \varphi^{*}, \quad \boldsymbol{B}^{*}=\nabla^{\prime} \times \boldsymbol{A}^{*} .
\end{aligned}
$$

It follows [14] from the assumption $(47)_{1},(48)_{1}$ and from the Gauss electric law (42) that

$$
\int_{\mathscr{G}^{\prime}}\left(\boldsymbol{D} \cdot \boldsymbol{E}-\boldsymbol{D}^{*} \cdot \boldsymbol{E}^{*}\right) d V^{\prime}=-\int_{\mathscr{G}^{\prime}} q_{f} \varphi d V^{\prime}-\oint_{\partial \mathscr{G}^{\prime}} \omega_{f} \varphi d A^{\prime}
$$

where $\boldsymbol{D}$ is the dielectric displacement vector $\left(\boldsymbol{D}=\varepsilon_{o} \boldsymbol{E}+\boldsymbol{P}\right.$ in $\mathscr{G}^{\prime}$ and $\boldsymbol{D}^{*}=\varepsilon_{o} \boldsymbol{E}^{*}$ in $\left.\mathscr{G}^{\prime} \cup \mathscr{G}^{*}\right) ; q_{f}$ is the free electric charge distribution in $\mathscr{G}^{\prime}$ and $\omega_{f}$ is the surface free electric charge distribution on $\partial \mathscr{G}^{\prime}$ (there is no free electric charge in $\mathscr{G}^{*}$ ). Moreover, it is assumed that $\varphi=\varphi^{*}$ on $\partial \mathscr{G}^{\prime}$. Taking (49) into account, the functional (45) can be written in the form

$$
L=-\int_{\mathscr{G}^{\prime}}\left[\rho\left(\psi^{(*)}+\tilde{g}+\tilde{\lambda}\right)-\left(\mathbf{M} e-\mathbf{M} e^{*}\right)-q_{f} \varphi\right] d V^{\prime}+\oint_{\partial \mathscr{G}^{\prime}} \omega_{f} \varphi d A^{\prime}
$$

where $\psi^{(*)}$ is the thermodynamic function defined by

$$
\psi^{(*)} \doteq \epsilon-T^{*} \eta-\frac{1}{\rho} \boldsymbol{P} \cdot \boldsymbol{E}
$$

and

$$
\begin{aligned}
& \mathbf{M} e \doteq \frac{1}{2}\left(\varepsilon_{o} \boldsymbol{E} \cdot \boldsymbol{E}-\frac{1}{\mu_{o}} \boldsymbol{B} \cdot \boldsymbol{B}\right) \\
& \mathbf{M} e^{*} \doteq \frac{1}{2}\left(\varepsilon_{o} \boldsymbol{E}^{*} \cdot \boldsymbol{E}^{*}-\frac{1}{\mu_{o}} \boldsymbol{B}^{*} \cdot \boldsymbol{B}^{*}\right) .
\end{aligned}
$$

The quantity $\psi^{(*)}$ is a function depending on the set of independent variables. In the case where the liquid crystal is in a thermodynamic equilibrium state, $\psi^{(*)}$ has the form [7], [11]

$$
\psi^{(*)}=\psi^{(*)}\left(\rho^{-1}, \mathbf{j}, \boldsymbol{\gamma}, T, \boldsymbol{E}, \boldsymbol{B}\right),
$$


where $\mathbf{j}$ is the microinertia tensor, $\boldsymbol{\gamma}$ is the wryness deformation tensor and $T$ is the thermostatic temperature of the liquid crystal. It is assumed in the variational approach [11] that the variations $\delta \boldsymbol{x}^{\prime}, \delta \phi^{*}, \delta \boldsymbol{A}^{*}$ are the independent and continuous functions in the whole space $V ; \delta \phi, \delta \boldsymbol{A}$ are the independent and continuous functions in $\mathscr{G}^{\prime}$ and $\delta \phi=\delta \phi^{*}, \delta \boldsymbol{A}=\delta \boldsymbol{A}^{*}$ on $\partial \mathscr{G}^{\prime}$. For micropolar media we define in $\mathscr{G}^{\prime}$ the angular variation $\delta \boldsymbol{\kappa}$ by the equation below [13], [11]:

$$
\delta \boldsymbol{\xi}=\delta \boldsymbol{\kappa} \times \xi .
$$

The fundamental assumption of the variational approach [13]

$$
\delta \boldsymbol{X}=\mathbf{0},
$$

allows us to write the following important formulae

$$
\begin{aligned}
\delta \rho^{-1} & =\rho^{-1} \nabla^{\prime} \cdot \delta \boldsymbol{x}^{\prime}, \\
\delta \mathbf{j} & =\mathbf{j} \cdot \boldsymbol{\varepsilon} \cdot \delta \boldsymbol{\kappa}-\delta \boldsymbol{\kappa} \cdot \boldsymbol{\varepsilon} \cdot \mathbf{j}, \\
\delta \gamma & =-\gamma \cdot\left(\nabla^{\prime} \otimes \delta \boldsymbol{x}^{\prime}\right)^{T}+\left(\nabla^{\prime} \otimes \delta \boldsymbol{\kappa}\right)^{T}+\boldsymbol{\varepsilon}:(\delta \boldsymbol{\kappa} \otimes \gamma), \\
\delta \boldsymbol{E} & =\nabla^{\prime}(\delta \varphi)-\boldsymbol{E} \cdot\left(\nabla^{\prime} \otimes \delta \boldsymbol{x}^{\prime}\right)^{T}, \\
\delta \boldsymbol{B} & =\nabla^{\prime} \times \hat{\delta} \boldsymbol{A}+\left(\boldsymbol{B} \cdot \nabla^{\prime}\right) \delta \boldsymbol{x}^{\prime}-\boldsymbol{B}\left(\nabla^{\prime} \cdot \delta \boldsymbol{x}^{\prime}\right), \\
\delta \boldsymbol{E}^{*} & =\nabla^{\prime}\left(\delta \varphi^{*}\right)-\boldsymbol{E}^{*} \cdot\left(\nabla^{\prime} \otimes \delta \boldsymbol{x}^{\prime}\right)^{T}, \\
\delta \boldsymbol{B}^{*} & =\nabla^{\prime} \times \hat{\delta} \boldsymbol{A}^{*}+\left(\boldsymbol{B}^{*} \cdot \nabla^{\prime}\right) \delta \boldsymbol{x}^{\prime}-\boldsymbol{B}^{*}\left(\nabla^{\prime} \cdot \delta \boldsymbol{x}^{\prime}\right),
\end{aligned}
$$

where the Weiss gauge invariant variations $\hat{\delta} \boldsymbol{A}\left(\hat{\delta} \boldsymbol{A}^{*}\right)$ are defined by

$$
\hat{\delta} \boldsymbol{A} \doteq \delta \boldsymbol{A}-\left(\nabla^{\prime} \otimes \boldsymbol{A}\right)^{T} \cdot \delta \boldsymbol{x}^{\prime}, \quad \hat{\delta} \boldsymbol{A}^{*} \doteq \delta \boldsymbol{A}^{*}-\left(\nabla^{\prime} \otimes \boldsymbol{A}^{*}\right)^{T} \cdot \delta \boldsymbol{x}^{\prime} .
$$

Moreover,

$$
\begin{aligned}
& \delta \int_{\mathscr{G}^{\prime}} \rho(\tilde{g}+\tilde{\lambda}) d V^{\prime}=-\int_{\mathscr{G}^{\prime}} \rho\left(\tilde{\boldsymbol{f}} \cdot \delta \boldsymbol{x}^{\prime}+\tilde{\boldsymbol{l}} \cdot \delta \boldsymbol{\kappa}\right) d V^{\prime} \\
& \delta \int_{\mathscr{G}^{\prime}}^{q_{f}} \varphi d V^{\prime}+\delta \oint_{\partial \mathscr{G}^{\prime}} \omega_{f} \varphi d A^{\prime}=\int_{\mathscr{G}^{\prime}} q_{f} \delta \varphi d V^{\prime}+\oint_{\partial \mathscr{G}^{\prime}} \omega_{f} \delta \varphi d A^{\prime} .
\end{aligned}
$$

The last formula follows from the Gauss electric law, which states that

$$
\int_{\mathscr{G}^{\prime}} q_{f} d V^{\prime}+\oint_{\partial \mathscr{G}^{\prime}} \omega_{f} d A^{\prime}=0
$$

Taking into account the last expressions, the variational problem (46) takes the form

$$
\begin{aligned}
& \int_{\mathscr{G}^{\prime}}\left[\rho\left(-\delta \psi^{(*)}+\tilde{\boldsymbol{f}} \cdot \delta \boldsymbol{x}^{\prime}+\tilde{\boldsymbol{l}} \cdot \delta \boldsymbol{\kappa}\right)+\delta\left({ }_{\mathbf{M}} e-\mathbf{M} e^{*}\right)\right. \\
& \left.\left.\quad+{ }_{\mathbf{M}} e-{ }_{\mathbf{M}} e^{*}\right) \nabla^{\prime} \cdot \delta \boldsymbol{x}^{\prime}+q_{f} \delta \varphi\right] d V^{\prime}+\oint_{\partial \mathscr{G}^{\prime}} \omega_{f} \delta \varphi d A^{\prime}=0,
\end{aligned}
$$


where

$$
\delta \psi^{(*)}=\frac{\partial \psi^{(*)}}{\partial \boldsymbol{y}} \cdot \delta \boldsymbol{y},
$$

and

$$
\boldsymbol{y} \doteq\left(\rho^{-1}, \mathbf{j}, \gamma, T, \boldsymbol{E}, \boldsymbol{B}\right)
$$

We introduce the following constitutive relations [7]

$$
\begin{aligned}
& \pi=-\frac{\partial \psi^{(*)}}{\partial \rho^{-1}}, \quad \boldsymbol{m}=\rho \frac{\partial \psi^{(*)}}{\partial \boldsymbol{\gamma}^{T}}, \quad \boldsymbol{t}=-\pi \boldsymbol{I}-\boldsymbol{m} \cdot \boldsymbol{\gamma}, \\
& \boldsymbol{P}=-\rho \frac{\partial \psi^{(*)}}{\partial \boldsymbol{E}}, \quad \boldsymbol{M}=-\rho \frac{\partial \psi^{(*)}}{\partial \boldsymbol{B}},
\end{aligned}
$$

where $\pi$ is the thermostatic pressure and $\boldsymbol{t}$ and $\boldsymbol{m}$ are the equilibrium stress and couple stress tensors, respectively.

Making use of the constitutive relations (64), equations (57), the material objectivity and the assumption of the continuity of the variations, we obtain the full system of electro-magneto-statics equations for liquid crystals in case of thermodynamic equilibrium:

$$
\begin{aligned}
& \nabla^{\prime} \cdot \boldsymbol{t}+{ }_{\mathbf{M}} \boldsymbol{f}+\rho \tilde{\boldsymbol{f}}=\nabla^{\prime} \cdot{ }_{\mathbf{M}} \boldsymbol{t}^{*}=\mathbf{0}, \\
& \nabla^{\prime} \cdot \boldsymbol{m}+\boldsymbol{\varepsilon}: \boldsymbol{t}+{ }_{\mathbf{M}} \boldsymbol{l}+\rho \tilde{\boldsymbol{l}}=\mathbf{0}, \\
& \nabla^{\prime} \cdot \boldsymbol{D}=q_{f}, \quad \varepsilon_{o} \nabla^{\prime} \cdot \boldsymbol{E}^{*}=0, \\
& \nabla^{\prime} \times \boldsymbol{H}=\mathbf{0}, \quad \nabla^{\prime} \times \boldsymbol{B}^{*}=\mathbf{0},
\end{aligned}
$$

with the boundary conditions on $\partial \mathscr{G}^{\prime}$

$$
\begin{aligned}
& \boldsymbol{n}^{\prime} \cdot(\boldsymbol{t}-\llbracket \mathbf{M} \boldsymbol{t} \rrbracket)=\mathbf{0}, \\
& \boldsymbol{n}^{\prime} \cdot \boldsymbol{m}=\mathbf{0}, \\
& \boldsymbol{n}^{\prime} \cdot \llbracket \boldsymbol{D} \rrbracket=\omega_{f}, \\
& \boldsymbol{n}^{\prime} \times \llbracket \boldsymbol{H} \rrbracket=\mathbf{0},
\end{aligned}
$$

where ${ }_{\mathbf{M}} \boldsymbol{f}=\nabla^{\prime} \cdot{ }_{\mathbf{M}} \boldsymbol{t},{ }_{\mathbf{M}} \boldsymbol{l}=\boldsymbol{\varepsilon}:{ }_{\mathbf{M}} \boldsymbol{t}^{T}$ and

$$
\begin{aligned}
{ }_{\mathbf{M}} \boldsymbol{t} & =\boldsymbol{D} \otimes \boldsymbol{E}+\boldsymbol{B} \otimes \boldsymbol{H}-\frac{1}{2}[(\boldsymbol{D}-\boldsymbol{P}) \cdot \boldsymbol{E}+\boldsymbol{B} \cdot(\boldsymbol{H}-\boldsymbol{M})] \boldsymbol{I} \\
\mathbf{M}^{*} & =\varepsilon_{o} \boldsymbol{E}^{*} \otimes \boldsymbol{E}^{*}+\frac{1}{\mu_{o}} \boldsymbol{B}^{*} \otimes \boldsymbol{B}^{*}-{ }_{\mathbf{M}} \epsilon^{*} \boldsymbol{I} .
\end{aligned}
$$


Besides the equations (65)-(68) we obtain the additional equation (if $\delta T \neq 0$ )

$$
\frac{\partial \psi^{(*)}}{\partial T}=0
$$

If we introduce now the Helmholtz free energy function $\psi$ in the usual form [7], [10]:

$$
\psi=\epsilon-T \eta-\frac{1}{\rho} \boldsymbol{P} \cdot \boldsymbol{E},
$$

then we obtain

$$
\psi^{(*)}=\psi+\left(T-T^{*}\right) \eta
$$

The free energy $\psi$ is the potential for entropy, i.e.

$$
\eta=-\frac{\partial \psi}{\partial T}
$$

Taking into account equations (72)-(74) we obtain from (71) and (64) the following restrictions

$$
\left(T-T^{*}\right) \frac{\partial \eta}{\partial T}=0
$$

Thus it is derived that in equilibrium the thermostatic temperature of the liquid crystal has to be equal to the temperature of the environment $\left(T=T^{*}\right)$.

\section{Conclusions}

Starting out with the First and Second Law of Thermodynamics, with Maxwell's equations and with some additional presuppositions, an evolution criterion far from equilibrium can be derived. It states, that a certain global property of a non-isolated system contacted by an equilibrium environment is always increasing over time. This global property is a modified electromagnetic free energy. The additional restrictions are that the equilibrium environment is black-body radiation, that the force density is conservative and that the exchange between the considered system and its environment is purely thermal. Consequently, the well-known evolution criterion is extended to the electromagnetic case.

In equilibrium the evolution criterion changes into a variational problem resulting in differential equations describing the considered system. Here these equations are applied to liquid crystals in electromagnetic fields which are described by Eringen's approach for micropolar media.

Taking into account the constitutive relation (54) in the form proper for another micropolar medium, e.g. for the elastic micropolar medium or the micropolar fluid 
(see [9], [15]), one can obtain the equations describing the thermodynamical equilibrium process in that type of material. We conclude that the functional

$$
L \doteq \int_{\mathscr{G}(t)}\left[\rho\left(T^{*} \eta-\epsilon-\mathbf{k} e-\tilde{g}-\tilde{\lambda}\right)-\left(\mathbf{M} \epsilon-\mathbf{M} \epsilon^{*}\right)\right] d V
$$

is not decreasing for any thermodynamic process occurring in an arbitrary electromagnetic body and fulfilling the assumptions discussed above about the interaction of the system with the environment. That functional $L$ attains the maximum for the thermodynamical equilibrium processes. In that sense the inequality (34) is an evolution criterion.

\section{Nomenclature}

\section{Latin letters}

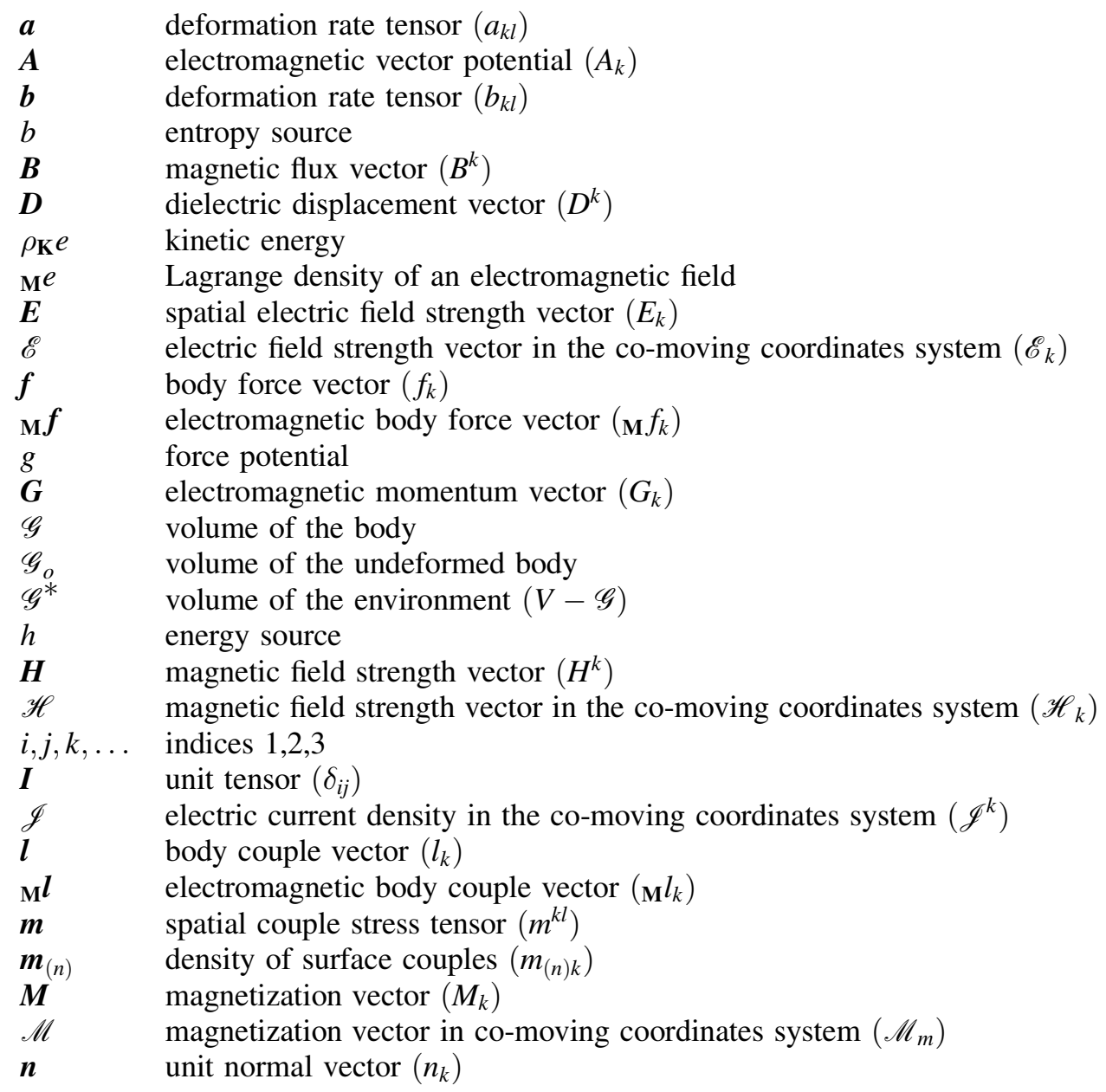




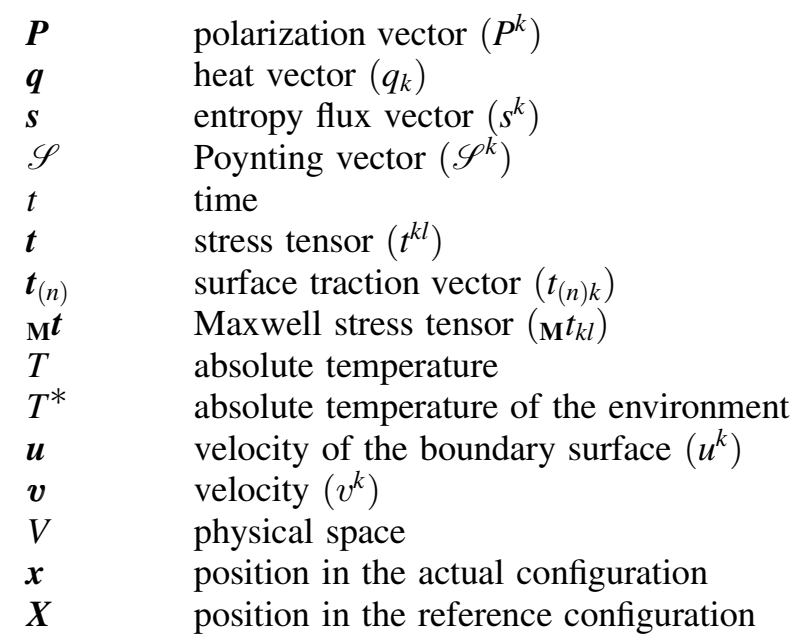

German letters

$\mathfrak{c} \quad$ Cosserat deformation tensor in the Euler description $\left(\mathfrak{c}_{\mathrm{fl}}\right)$

$\mathfrak{c}$ Cosserat deformation tensor in the Lagrange description $\left(\mathfrak{C}_{K L}\right)$

j spatial microinertia tensor $\left(j^{k l}\right)$

\section{Greek letters}

$\begin{array}{ll}\gamma & \text { spatial wryness deformation tensor }\left(\gamma_{k l}\right) \\ \Gamma & \text { material wryness deformation tensor }\left(\Gamma_{K L}\right) \\ \epsilon & \text { internal energy density } \\ \mathbf{M} \epsilon & \text { quantity of the dimension of the electromagnetic energy } \\ \varepsilon_{o} & \text { electric permittivity of vacuum } \\ \boldsymbol{\varepsilon} & \text { alternating tensor }\left(\varepsilon_{i j k}\right) \\ \eta & \text { entropy density } \\ \lambda & \text { couple force potential } \\ \mu_{o} & \text { magnetic permeability of vacuum } \\ \omega & \text { angular velocity vector }\left(\omega^{k}\right) \\ \pi & \text { thermostatic pressure } \\ \rho & \text { mass density of a body in the actual state } \\ \rho_{o} & \text { mass density of a body in the reference state } \\ \boldsymbol{\sigma} & \text { spin density vector }\left(\sigma^{k}\right) \\ \varphi & \text { electromagnetic scalar potential } \\ \psi & \text { Helmholtz free energy function } \\ \xi & \text { director in the actual configuration }\left(\xi^{k}\right) \\ & \text { director in the material configuration }\left(\Xi^{K}\right)\end{array}$

Other symbols

$d A \quad$ surface element

$d V \quad$ volume element 
$\begin{array}{ll}\partial & \text { boundary of a region } \\ \nabla & \text { nabla operator }\left(\partial_{k}\right) \\ \frac{D}{D t} & \text { material time derivative } \\ \frac{D^{*}}{D t} & \text { convective derivative } \\ \boldsymbol{F}^{*} & \text { convective derivative of the vector } \boldsymbol{F} \\ \dot{F} & \text { material time derivative of the quantity } F \\ \widetilde{F} & \text { given quantity, describes applied field } F \\ \mathbf{M} F & \text { electromagnetic type quantity } F \\ \llbracket F \rrbracket & \text { jump of the quantity } F \text { on the discontinuity surface } \partial \mathscr{G}\end{array}$

\section{References}

[1] Glansdorff, P., Prigogine, I., On a general evolution criterion in macroscopic physics, Physica, 30 (1964), 351-374.

[2] Glansdorff, P., Prigogine, I., Non-equilibrium stability theory, Physica, 46 (1970), 344 366.

[3] Glansdorff, P., Prigogine, I., Thermodynamic Theory of Structure, Stability and Fluctuations, Wiley, London, 1971.

[4] De Groot, S. R., Mazur, P., Non-Equilibrium Thermodynamics, North-Holland, Amsterdam, 1962.

[5] Muschik, W., Papenfuss, C., An evolution criterion of nonequilibrium thermodynamics and its application to liquid crystals, Physica A, 201 (1993), 515-526.

[6] Papenfuss, C., Muschik, W., Evolution criterion in nonequilibrium thermodynamics and a variational principle for equilibrium states of free-standing liquid crystal films, Physical Review E, 56 (1997), 4275-4281.

[7] Eringen, A. C., Continuum theory of nematic liquid crystals subject to electromagnetic fields, J. Math. Phys., 20 (1979), 2671-2681.

[8] Eringen, A. C., Kafadar, C. B., Polar field theories, in: Continuum Physics, Vol. 4, Ed. A. C. Eringen, Academic Press, New York, 1976.

[9] Eringen, A. C., Microcontinuum Field Theories, Vol. 1, Springer, New York, 1998.

[10] Eringen, A. C., Maugin, G. A., Electrodynamics of Continua, Vol. 1, Springer Verlag, New York, 1990.

[11] Kotowski, R., Radzikowska, E., Variational approach to the thermo-electrodynamics of liquid crystals, Int. J. Engng. Sci., 37 (1999), 771-802.

[12] Liggieri, L., Sanfeld, A., Steinchen, A., Effects of magnetic and electric fields on surface tension of liquids, Physica A, 206 (1994), 299-331.

[13] Kotowski, R., Radzikowska, E., Variational principle in thermo-electromagneto-dynamics of micropolar media, J. Techn. Phys., 39 (1998), 283-295.

[14] Ingarden, R. S., Jamiotkowski, A., Classical Electrodynamics, Elsevier-PWN, Amsterdam, Warszawa, 1985.

[15] Kotowski, R., Radzikowska, E., Variational principle for dissipative processes in continuum physics, Int. J. Engng. Sci., 38 (2000), 1129-1149.

Paper received: 2000-06-16

Paper accepted: 2001-02-27

Dr. E. Radzikowska ${ }^{2}$

Dr. R. Kotowski

Institute of Fundamental Technological Research

${ }^{2)}$ Corresponding author: eradzik@ippt.gov.pl 
Polish Academy of Sciences

Świętokrzyska 11/21

00-049 Warsaw

Poland

E-mail: eradzik@ippt.gov.pl

E-mail: rkotow@ippt.gov.pl

Dr. R. Kotowski

Polish-Japanese Institute for Information Technology

Koszykowa 86

02-008 Warsaw

Poland

E-mail: rkotow@pjwstk.waw.pl

Prof. Dr. W. Muschik

Institut für Theoretische Physik

Technische Universität Berlin

Hardenbergstrasse 36

10623 Berlin

Germany

E-mail: muschik@physik.tu-berlin.de 